\title{
GW23-e1018 UP-REGULATING H0-1 IMPROVES POST-INFARCTION HEART FUNCTION OF SPONTANEOUS HYPERTENSIVE RATS VIA ANTI-INFLAMMATION, ANTI-OXIDATION, LOWERING BLOOD GLUCOSE AND IMPROVING ENDOTHELIAL FUNCTION
}

doi:10.1136/heartjnl-2012-302920a.4

Jian Cao, Jian Cao. First Geriatric Cardiology Department of South Building, Chinese PLA General Hospital

Objectives Our previous results showed that hame oxygenase 1 $(\mathrm{HO}-1)$ is involved in the regulation of endothelial function by the modulation of NOS isoforms and control of oxidative stress. HO-1, in particular, may be well suited as a therapeutic agent for myocardial protection, because the catabolic by-products of hame metabolism, carbon monoxide (CO) and bilirubin, have been reported to exert pleiotropic cytoprotective effects, including inhibition of oxidative stress, inflammation, and apoptosis.

Methods Male spontaneous hypertensive rats (SHR) at 13 weeks $(n=40)$ and age-matched male Wistar rats $(n=20)$ were selected. After basal echocardiography and blood pressure measurement, they were divided into six groups: (1) WT (sham +NS), (2) WT (sham +Copp), (3) SHR(MI+NS), (4) SHR (MI+Copp), (5) SHR (MI + Copp+SnMP), (6) SHR (sham+NS), n=10/group. Sham operation or coronary ligation were performed respectively. The first day after operation, medications were administered among groups: normal saline (NS), or cobalt protoporphyrin (CoPP), an inducer of $\mathrm{HO}-1,4.5 \mathrm{mg} / \mathrm{kg}$, or concurrently with tin mesoporphyrin IX dichloride (SnMP), an inhibitor of $\mathrm{HO}$ activity, $15 \mathrm{mg} / \mathrm{kg}$, all for 6 weeks, 1/week, intraperitoneally. At 6 weeks, trans-thoracic echocardiography and hemodynamics tests was performed; thereafter, blood was collected for blood biochemistry, $\mathrm{NO}, \mathrm{PGI}_{2}$ testing. One part of isolated hearts were fixed into $10 \%$ formalin, paraffin embedded, processed for HE and Masson's trichrome stain. The other part of the hearts were rapidly frozen in liquid nitrogen, stored at $-80^{\circ} \mathrm{C}$, prepared for western blot analysis of $\mathrm{HO}-1$ expression.

\section{Results}

(1) Blood pressure: Compared with WTs, SHRs exhibited significantly higher blood pressure, including systolic blood pressure (SBP) (212.371 \pm 37.309 vs 138.081 $\pm 13.057, \mathrm{p}=0.000)$, diastolic blood pressure (DBP) $(193.545 \pm 29.374$ vs $115.795 \pm 19.305, p=0.000)$, and mean arterial pressure (MAP) $(205.002 \pm 40.234$ vs 123.223 $\pm 17.039, \mathrm{p}=0.000$ ). Copp treatment significantly lowered SBP, DBP, MAP in SHR(MI+Copp) compared to SHR(MI+NS) (SBP: 154.753 $\pm 10.591, p=0.000$; DBP: $136.900 \pm 12.392, p=0.000 ;$ MAP: $142.851 \pm 11.622, \mathrm{p}=0.000)$

(2) Cardiac function: Left ventricular ejection fraction (LVEF) and left ventricular fraction shortening (LVFS) between WTs and SHRs before operation are similar. After coronary ligation operation on SHRs, LVEF and LVFS were significantly decreased in SHR 
$(\mathrm{MI}+\mathrm{NS})$ group as compared with WT (sham+NS) group. Besides, +dp/dtmax and (-dp/dtmax)/1/VSP were also significantly decreased in SHR (MI+NS) group. Copp treatment could improve LVEF (72.050 \pm 2.681 vs $55.967 \pm 7.340, p=0.000)$, LVFS (35.975 \pm 2.012 vs $25.500 \pm 4.299, \mathrm{p}=0.000),+\mathrm{dp} / \mathrm{dtmax}(2591.92$ \pm 53.85 vs $1375.29 \pm 266.13, \quad p=0.000), \quad(-d p / d t m a x) / 1 / V S P$ $(-17.44 \pm 0.32$ vs $-9.95 \pm 0.11, p=0.000)$ in SHR (MI+Copp), while decrease LVEDD $(0.4725 \pm 0.0985$ vs $0.6411 \pm 0.0903$, $\mathrm{p}=0.001)$ and LVESD $(0.3025 \pm 0.0675$ vs $0.4778 \pm 0.0748$, $\mathrm{p}=0.000)$ as compared with SHR(MI+NS).

(3) Blood parameters: SHR (MI+NS) group exhibited higher CRP levels, lower NO levels as compared with WT (sham+NS) group, while the levels of $\mathrm{TB}, \mathrm{Glu}$, and $\mathrm{PGI}_{2}$ were similar between two groups. Copp treatment could lower CRP (0.090 \pm 0.010 vs $0.142 \pm 0.036, p=0.020)$ and Glu $(5.560 \pm 1.277$ vs $7.620 \pm 0.896$, $\mathrm{p}=0.001)$ levels, and elevate TB $(1.673 \pm 0.188$ vs $1.036 \pm 0.426$, $\mathrm{p}=0.033)$, NO $(30.915 \pm 5.853$ vs $19.483 \pm 2.967, p=0.001)$, $\mathrm{PGI}_{2}(4.361 \pm 0.991$ vs $1.901 \pm 0.801, \mathrm{p}=0.000)$ levels in SHR (MI+Copp) group as compared with SHR(MI+NS).

(4) Histology: SHR (MI+NS) group exhibited obvious myocardial infarction, LV fibrosis in infarct area; and also showed myocyte hypertrophy, hyperaemia, and chronic inflammation in peri-infarct area. Besides, HW/BW ratio was also significantly higher in SHR $(\mathrm{MI}+\mathrm{NS})$ group as compared with WT $($ sham $+\mathrm{NS})$ group. Copp treatment could significantly diminish infarct area, lower the degree of fibrosis, inhibit myocyte hypertrophy, hyperaemia, and chronic inflammation in peri-infarct area, and decrease the ratio of HW/BW $(0.382 \pm 0.014$ vs $0.472 \pm 0.065, p=0.001)$ compared with $\mathrm{SHR}(\mathrm{MI}+\mathrm{NS})$.

Western blot: Copp treatment could elevate HO-1 expression (HO-1/Tubulin $1.065 \pm 0.061$ vs $0.930 \pm 0.059, \mathrm{p}=0.019$ ) in SHR (MI+Copp) group as compared with SHR (MI+NS) group, while concurrently with SnMP could not only block the up-regulation of HO-1 expression, but also inhibit the accompanied amelioration of blood pressure and cardiac function in SHR with MI.

Conclusions Spontaneous hypertensive rats with myocardial infarction exhibited high blood pressure, impaired systolic and diastolic cardiac function, myocyte hypertrophy, heart dilation, excessive inflammation, and glucose metabolic and endothelial dysfunction. Up-regulation of $\mathrm{HO}-1$ via Copp treatment could lower blood pressure, decrease infarct area, improve post-infarct cardiac function, and inhibit ventricular remodelling in SHR with MI. The relative mechanism might involve inhibiting inflammation, anti-oxidation, improving glucose metabolism and endothelial function. 